\title{
Solvability of $F_{4}$ quantum integrable systems*
}

\author{
Juan C. Lopez Vieyra ${ }^{\dagger}$ and Alexander V. Turbiner $\ddagger \S$ \\ Instituto de Ciencias Nucleares, UNAM, A.P. 70-543, 04510 México D.F., México
}

\begin{abstract}
It is shown that the $F_{4}$ rational and trigonometric integrable systems are exactly-solvable for arbitrary values of the coupling constants. Their spectra are found explicitly while eigenfunctions are obtained by pure algebraic means. For both systems new variables are introduced in which the Hamiltonian has an algebraic form being also (block)-triangular. These variables are a certain invariants of the $F_{4}$ Weyl group. Both Hamiltonians preserve the same (minimal) flag of spaces of polynomials, which is found explicitly.
\end{abstract}

pacs: $02.30 . \mathrm{Ik}, 02.20 . \mathrm{Sv}, 02.70 . \mathrm{Hm}$

keywords: Quantum Integrable systems, Exact solvability, Root space invariants

\section{Introduction}

The $F_{4}$ rational and trigonometric models (see e.g. [1]) belong to a family of the Hamiltonian systems originally found in the Hamiltonian reduction method introduced in the late seventies by Olshanetsky and Perelomov [2]. Their structure is related to the root systems of the $F_{4}$ algebra. There exist both classical and quantum $F_{4}$ systems and they are completely integrable.

In general, the Olshanetsky-Perelomov Hamiltonians possess different symmetries (permutations, translation-invariance, reflections, periodicity etc.). The idea of our approach is to code all these symmetries in new coordinates and hence study operators which can be called 'premature' and which give rise to these Hamiltonians. It was formulated for the first time in [3], where $A_{n}$ rational and trigonometric models (or, in other words, Calogero and Sutherland models) were studied. Later this approach was developed for $G_{2}$ rational and trigonometric models [4. It is almost evident that above-mentioned variables

\footnotetext{
*Talk given by JCLV at the 12th INTERNATIONAL COLLOQUIUM Quantum Group and Integrable Systems, Prague, 12-14 June, 2003

†vieyra@nuclecu.unam.mx

¥turbiner@nuclecu.unam.mx

$\S$ On leave of absence from the Institute for Theoretical and Experimental Physics, Moscow 117259, Russia.
} 
are nothing but a certain Weyl-invariant functions with an extra property of periodicity in trigonometric case. We will show that taking for $F_{4}$ quantum Hamiltonians suitable invariants of the $F_{4}$ Weyl group as the variables we arrive at the rational and trigonometric Hamiltonians in an algebraic form, when the coefficient functions in front of derivatives are polynomials. This property uncovers a hidden algebra of both models and explains the exact solvability of the $F_{4}$ quantum systems. Mostly, present Talk is based on the article [1]. The results presented below appeared as a conclusion of about five years of the intense study of the $F_{4}$ quantum systems.

\section{$2 \quad F_{4}$-Rational model}

The $F_{4}$-rational model describes a quantum particle in four-dimensional space with a Hamiltonian given by

$$
\begin{gathered}
H_{F_{4}}^{(r)}=\frac{1}{2} \sum_{i=1}^{4}\left(-\partial_{i}^{2}+\omega^{2} x_{i}^{2}\right)+g \sum_{j>i}^{4}\left(\frac{1}{\left(x_{i}-x_{j}\right)^{2}}+\frac{1}{\left(x_{i}+x_{j}\right)^{2}}\right) \\
+g_{1} \sum_{i=1}^{4} \frac{1}{x_{i}^{2}}+4 g_{1} \sum_{\nu^{\prime} s=0,1}^{4} \frac{1}{\left[x_{1}+(-1)^{\nu_{2}} x_{2}+(-1)^{\nu_{3}} x_{3}+(-1)^{\nu_{4}} x_{4}\right]^{2}} .
\end{gathered}
$$

The form of the interactions is dictated by the set of positive simple roots of the $F_{4}$ algebra. This model contains two independent coupling constants, $g$ and $g_{1}$, each of them associated with roots of different lengths, and $\omega$ is the frequency of the harmonic oscillator term. The configuration space coincides to the $F_{4}$ Weyl chamber.

Now we describe the procedure of solving the spectral problem $H_{F_{4}}^{(r)} \psi=E \psi$ (see [1]).

1. At first let us remind the known form of the ground state eigenfunction

$$
\begin{aligned}
\Psi_{0}= & \prod_{j<i}\left(x_{i}+x_{j}\right)^{\nu} \prod_{j<i}\left(x_{i}-x_{j}\right)^{\nu} \prod_{i} x_{i}^{\mu} . \\
& \cdot \prod_{\nu^{\prime} s=0,1}^{4}\left(x_{1}+(-1)^{-\nu_{2}} x_{2}+(-1)^{-\nu_{3}} x_{3}+(-1)^{-\nu_{4}} x_{4}\right)^{\mu} e^{-\frac{\omega}{2} \sum x_{i}^{2}},
\end{aligned}
$$

where $g=\nu(\nu-1)>-\frac{1}{4}$, and $g_{1}=\frac{1}{2} \mu(\mu-1)>-\frac{1}{8}$.

2. A general feature of the quantum rational Hamiltonians stemming from the Hamiltonian reduction method is that all eigenfunctions admit a factorization in a form of the ground state eigenfunction $\Psi_{0}$ multiplied by a polynomial in Cartesian coordinates. Exploiting it we gauge away the ground state,

$$
h_{F_{4}}^{(r)}=2\left(\Psi_{0}\right)^{-1} H_{F_{4}}^{(r)} \Psi_{0}
$$


3. In order to try to find an algebraic form of the gauge rotated Hamiltonian (3) we make a change of variables

$$
\left(x_{1}, x_{2}, x_{3}, x_{4}\right) \rightarrow\left(t_{1}, t_{3}, t_{4}, t_{6}\right)
$$

where the new variables $t_{i}, i=1,3,4,6$ are the Weyl invariant polynomials with respect to the $F_{4}$ group of the lowest degrees (in our notations, the indices $i=1,3,4,6$ correspond to the degrees $2,6,8,12$ ).

The appropriate set of variables in which the problem takes an algebraic form is

$$
\begin{aligned}
t_{1} & =\sigma_{1}, \quad t_{3}=\sigma_{3}-\frac{1}{6} \sigma_{1} \sigma_{2}, \quad t_{4}=\sigma_{4}-\frac{1}{4} \sigma_{1} \sigma_{3}+\frac{1}{12} \sigma_{2}^{2}, \\
t_{6} & =\sigma_{4} \sigma_{2}-\frac{1}{36} \sigma_{2}^{3}-\frac{3}{8} \sigma_{3}^{2}+\frac{1}{8} \sigma_{1} \sigma_{2} \sigma_{3}-\frac{3}{8} \sigma_{1}^{2} \sigma_{4}
\end{aligned}
$$

where $\sigma_{n}=\sigma_{n}\left(x^{2}\right)$ are the elementary symmetric polynomials in variables $x_{i}^{2}, i=1,2,3,4$. In these variables the Hamiltonian becomes a second order differential operator

$$
h_{F_{4}}^{(r)}=\sum_{i \leq j}^{6} \mathcal{A}_{i j} \frac{\partial^{2}}{\partial t_{i} \partial t_{j}}+\sum_{i=1}^{6} \mathcal{B}_{i} \frac{\partial}{\partial t_{i}},
$$

with polynomial coefficients $\mathcal{A}_{i j}\left(=\mathcal{A}_{j i}\right), \mathcal{B}_{i}$ in variables $t_{1,3,4,6}$ of degrees $\leq 3$. Therefore, the operator $h_{F_{4}}^{(r)}$ is in the algebraic form we were looking for. Explicitly, the coefficients are

$$
\begin{gathered}
\mathcal{A}_{11}=2 t_{1}, \quad \mathcal{A}_{13}=6 t_{3}, \quad \mathcal{A}_{14}=8 t_{4}, \quad \mathcal{A}_{16}=12 t_{6} \\
\mathcal{A}_{33}=-\frac{1}{3} t_{3} t_{1}^{2}+\frac{10}{3} t_{4} t_{1}, \quad \mathcal{A}_{34}=-\frac{2}{3} t_{1}^{2} t_{4}+4 t_{6}, \quad \mathcal{A}_{36}=8 t_{4}^{2}-t_{1}^{2} t_{6} \\
\mathcal{A}_{44}=-2 t_{3} t_{4}-t_{1} t_{6}, \quad \mathcal{A}_{46}=-2 t_{1} t_{4}^{2}-3 t_{3} t_{6} \\
\mathcal{B}_{1}=2 \omega t_{1}+24\left(\nu+\mu+\frac{1}{6}\right), \quad \mathcal{B}_{3}=6 \omega t_{3}-2\left(\nu+\frac{\mu}{2}+\frac{1}{4}\right) t_{1}^{2} \\
\mathcal{B}_{4}=8 \omega t_{4}-6\left(\nu+\frac{1}{3}\right) t_{3}, \quad \mathcal{B}_{6}=12 \omega t_{6}-6\left(\nu+\frac{2}{3}\right) t_{1} t_{4} .
\end{gathered}
$$

It is easy to check that the Hamiltonian $h_{\mathrm{F}_{4}}^{(r)}$ has infinitely-many finitedimensional invariant subspaces

$$
\mathcal{P}_{n}^{\left(F_{4}\right)}=\left\langle t_{1}^{p_{1}} t_{3}^{p_{3}} t_{4}^{p_{4}} t_{6}^{p_{6}} \mid 0 \leq p_{1}+2 p_{3}+2 p_{4}+3 p_{6} \leq n\right\rangle, n=0,1, \ldots
$$

with the characteristic vector $\vec{f}=(1,2,2,3)$ formed from the weight factors in front of $p_{1}, p_{3}, p_{4}, p_{6}$ in the definition (8). These invariant subspaces form an infinite Flag

$$
\mathcal{P}_{0}^{\left(F_{4}\right)} \subset \mathcal{P}_{1}^{\left(F_{4}\right)} \ldots \subset \mathcal{P}_{n}^{\left(F_{4}\right)} \subset \ldots
$$


The operator $h_{\mathrm{F}_{4}}^{(r)}$ with respect to action on monomials $t_{1}^{p_{1}} t_{3}^{p_{3}} t_{4}^{p_{4}} t_{6}^{p_{6}}$ has upper triangular form. The energies are given by

$$
E_{p_{1}, p_{2}, p_{3}, p_{4}}=2\left(p_{1}+3 p_{2}+4 p_{3}+6 p_{4}+2+12 \mu+12 \nu\right) \omega, \quad p_{i}=0,1,2, \ldots
$$

in agreement with general formula given in 2. The spectrum does not depend on the coupling constants $g, g_{1}$ (except for the reference point of the energy), it is equidistant and it coincides with the 4-dimensional harmonic oscillator spectrum but with different degeneracy, $n=p_{1}+3 p_{2}+4 p_{3}+6 p_{4}$. Since $h_{\mathrm{F}_{4}}^{(r)}$ preserves the flag the calculation of eigenfunctions is a linear-algebra procedure. It is important to make the following remark. Let us define a general flag of spaces of polynomials made out of

$$
\mathcal{P}_{n}=\left\langle t_{1}^{p_{1}} t_{3}^{p_{3}} t_{4}^{p_{4}} t_{6}^{p_{6}} \mid 0 \leq p_{1}+\alpha_{3} p_{3}+\alpha_{4} p_{4}+\alpha_{6} p_{6} \leq n\right\rangle, n=0,1, \ldots
$$

with the characteristic vector $\vec{f}=\left(1, \alpha_{3}, \alpha_{4}, \alpha_{6}\right)$. It can be easily checked that the same operator written in different variables can preserve different flags. Among these flags it can exist some minimal flag. We call the flag minimal if the $\alpha$ 's are minimal. Now we proceed to search of the minimal flag if exists.

It is evident that the Weyl-invariant polynomials of fixed degrees $t_{1,3,4,6}$ are defined ambiguously, up to invariants of lower degrees

$$
\begin{aligned}
& t_{1} \rightarrow t_{1}, \\
& t_{3} \rightarrow t_{3}+A t_{1}^{3}, \\
& t_{4} \rightarrow t_{4}+B_{1} t_{1}^{4}+B_{2} t_{1} t_{3}, \\
& t_{6} \rightarrow t_{6}+C_{1} t_{1}^{6}+C_{2} t_{1}^{3} t_{3}+C_{3} t_{1}^{2} t_{4}+C_{4} t_{3}^{2},
\end{aligned}
$$

where $A, B, C$ are arbitrary numbers. Exploiting this ambiguity we were able to find many different algebraic forms for the $F_{4}$-rational Hamiltonian $h_{\mathrm{F}_{4}}^{(r)}$ which preserve different flags of polynomials. Here we present a partial list of characteristic vectors of such flags for the $F_{4}$-rational model

$$
\begin{array}{ccccc}
(1,2,2,3) & (1,3,3,5) & (1,5,5,8) & (1,6,6,9) & (1,6,7,10) \\
(1,2,3,4) & (1,4,4,6) & (1,5,5,9) & (1,6,6,10) & (1,7,7,11) \\
(1,2,3,5) & (1,4,4,7) & (1,5,7,9) & (1,6,6,11) & \ldots
\end{array}
$$

By comparison of the characteristic vectors it is easy to see that the minimal flag exists and is characterized by the vector $(1,2,2,3)$. This result is in variance with one obtained in [5] where it was stated that the minimal vector is $(1,2,3,5)$. We were able to 'trigonometrize' the variables leading to the minimal flag, getting them as a rational limit of a certain trigonometric Weyl-invariant functions for those the $F_{4}$ trigonometric Hamiltonian appears in an algebraic form (see below). It seems that this feature holds for other models. 


\section{$3 \quad F_{4}$-Trigonometric model}

The Hamiltonian of the $F_{4}$ trigonometric model describes a four-dimensional quantum system in a periodic potential in all four directions. It is given by

$$
\begin{aligned}
\mathcal{H}_{\mathrm{F}_{4}}^{(t)}(x) & =-\frac{1}{2} \sum_{i=1}^{4} \partial_{x_{i}}^{2}+2 g \beta^{2} \sum_{j>i}^{4}\left(\frac{1}{\sin ^{2} \beta\left(x_{i}-x_{j}\right)}+\frac{1}{\sin ^{2} \beta\left(x_{i}+x_{j}\right)}\right) \\
+ & 2 g_{1} \beta^{2}\left(\sum_{i=1}^{4} \frac{1}{\sin ^{2} 2 \beta x_{i}}+\sum_{\nu^{\prime} s=0,1}^{4} \frac{1}{\sin ^{2} \beta\left[x_{1}+(-1)^{\nu_{2}} x_{2}+(-1)^{\nu_{3}} x_{3}+(-1)^{\nu_{4}} x_{4}\right]}\right)
\end{aligned}
$$

where the parameter $1 / \beta$ has meaning of the period, $g, g_{1}$ are the coupling constants associated with roots of different length. Configuration space of the problem coincides with the $F_{4}$ Weyl alcove.

The corresponding ground state eigenfunction is given by

$$
\Psi_{0}^{(t)}(x, \beta)=\left\{\Delta_{+}(x, \beta) \Delta_{-}(x, \beta)\right\}^{\nu}\left\{\Delta_{0}(x, 2 \beta) \Delta(x, 2 \beta)\right\}^{\mu},
$$

where $g=\nu(\nu-1) / 2, g_{1}=\mu(\mu-1)$, and

$$
\begin{aligned}
& \Delta_{ \pm}(x, \beta)=\beta^{-6} \prod_{j<i}^{4} \sin \beta\left(x_{i} \pm x_{j}\right), \quad \Delta_{0}(x, 2 \beta)=\beta^{-4} \prod_{i=1}^{4} \sin 2 \beta x_{i} \\
& \Delta(x, 2 \beta)=\beta^{-8} \prod_{\nu^{\prime} s=0,1}^{4} \sin \beta\left[x_{1}+(-1)^{\nu_{2}} x_{2}+(-1)^{\nu_{3}} x_{3}+(-1)^{\nu_{4}} x_{4}\right],
\end{aligned}
$$

are trigonometric analogues of the Weyl determinants.

In order to solve the spectral problem we again gauge away the ground state and define the gauge-rotated Hamiltonian

$$
h_{\mathrm{F}_{4}}^{(t)}=-2\left(\Psi_{0}^{(t)}(x)\right)^{-1}\left(\mathcal{H}_{\mathrm{F}_{4}}^{(t)}-E_{0}\right)\left(\Psi_{0}^{(t)}(x)\right),
$$

Now we search for a change of variables

$$
\left(x_{1}, x_{2}, x_{3}, x_{4}\right) \rightarrow\left(\tau_{1}, \tau_{3}, \tau_{4}, \tau_{6}\right),
$$

in which the gauge-rotated Hamiltonian $h_{\mathrm{F}_{4}}^{(t)}$ may take an algebraic form. Similarly to what was done for the rational case we look for the Weyl-invariant functions with an extra property of periodicity in each $x$-direction. The solution we found [1] is surprisingly easy and is given by

$$
\begin{aligned}
& \tau_{1}=\tilde{\sigma}_{1}-\frac{2 \beta^{2}}{3} \tilde{\sigma}_{2}, \\
& \tau_{3}=\tilde{\sigma}_{3}-\frac{1}{6} \tilde{\sigma}_{1} \tilde{\sigma}_{2}-2 \beta^{2}\left(\tilde{\sigma}_{4}-\frac{1}{36} \tilde{\sigma}_{2}^{2}\right), \\
& \tau_{4}=\tilde{\sigma}_{4}-\frac{1}{4} \tilde{\sigma}_{1} \tilde{\sigma}_{3}+\frac{1}{12} \tilde{\sigma}_{2}^{2}, \\
& \tau_{6}=\tilde{\sigma}_{4} \tilde{\sigma}_{2}-\frac{1}{36} \tilde{\sigma}_{2}^{3}-\frac{3}{8} \tilde{\sigma}_{3}^{2}+\frac{1}{8} \tilde{\sigma}_{1} \tilde{\sigma}_{2} \tilde{\sigma}_{3}-\frac{3}{8} \tilde{\sigma}_{1}^{2} \tilde{\sigma}_{4},
\end{aligned}
$$


(cf. (5) ) where $\tilde{\sigma}_{n}=\sigma_{n}\left(\frac{\sin ^{2}(\beta x)}{\beta^{2}}\right)$ are elementary symmetric polynomials of the periodic arguments. It is clear that $\tau_{1,3,4,6}$ are the Weyl invariant trigonometric (periodic) variables. In these variables the Hamiltonian becomes a second order differential operator with polynomial coefficients

$$
h_{\mathrm{F}_{4}}^{(t)}=\sum_{a<b} A_{a b} \frac{\partial^{2}}{\partial \tau_{a} \partial \tau_{b}}+\sum_{a}\left(B_{a}+C_{a}\right) \frac{\partial}{\partial \tau_{a}}, \quad a, b=1,3,4,6 .
$$

where

$$
\begin{aligned}
A_{11} & =4 \tau_{1}-4 \beta^{2} \tau_{1}^{2}-\frac{32}{3} \beta^{4} \tau_{3}-\frac{128}{9} \beta^{6} \tau_{4}, \\
A_{13} & =12 \tau_{3}-\frac{8}{3} \beta^{2}\left(4 \tau_{1} \tau_{3}+\tau_{4}\right)-\frac{32}{9} \beta^{4} \tau_{1} \tau_{4}, \\
A_{14} & =16 \tau_{4}-\frac{40}{3} \beta^{2} \tau_{1} \tau_{4}-\frac{16}{3} \beta^{4} \tau_{6}, \\
A_{16} & =24 \tau_{6}-20 \beta^{2} \tau_{1} \tau_{6}-\frac{32}{3} \beta^{4} \tau_{4}^{2}, \\
A_{33} & =-\frac{2}{3} \tau_{1}^{2} \tau_{3}+\frac{20}{3} \tau_{1} \tau_{4}-\frac{8}{9} \beta^{2}\left(18 \tau_{3}^{2}+\tau_{1}^{2} \tau_{4}+12 \tau_{6}\right), \\
A_{34} & =-\frac{4}{3} \tau_{1}^{2} \tau_{4}+8 \tau_{6}-\frac{4}{3} \beta^{2}\left(\tau_{1} \tau_{6}+12 \tau_{3} \tau_{4}\right), \\
A_{36} & =16 \tau_{4}^{2}-2 \tau_{1}^{2} \tau_{6}-\frac{8}{3} \beta^{2}\left(9 \tau_{3} \tau_{6}+\tau_{1} \tau_{4}^{2}\right), \\
A_{44} & =-4 \tau_{3} \tau_{4}-2 \tau_{1} \tau_{6}-24 \beta^{2} \tau_{4}^{2}, \\
A_{46} & =-4 \tau_{1} \tau_{4}^{2}-6 \tau_{3} \tau_{6}-36 \beta^{2} \tau_{4} \tau_{6}, \\
A_{66} & =-12 \tau_{3} \tau_{4}^{2}-6 \tau_{1} \tau_{4} \tau_{6}-8 \beta^{2}\left(6 \tau_{6}^{2}+\tau_{4}^{3}\right), \\
B_{1} & =8-8 \beta^{2} \tau_{1}, \\
B_{4} & =-4 \tau_{3}-\frac{88}{3} \beta^{2} \tau_{4}, \quad B_{6}=-8 \tau_{1} \tau_{4}-56 \beta^{2} \tau_{6}, \\
C_{1} & =48(\nu+\mu)-8 \beta^{2}(5 \nu+6 \mu) \tau_{1} \\
C_{3} & =-2(2 \nu+\mu) \tau_{1}^{2}-16 \beta^{2}(3 \nu+5 \mu) \tau_{3} \\
C_{4} & =-12 \nu \tau_{3}-24 \beta^{2}(3 \nu+4 \mu) \tau_{4} \\
C_{6} & =-12 \nu \tau_{1} \tau_{4}-48 \beta^{2}(2 \nu+3 \mu) \tau_{6}
\end{aligned}
$$

Similar to the rational case the coefficients $A_{i j}$ and $B_{i}, C_{i}$ are polynomials in $\tau$ 's of the degrees not higher than three and two, correspondingly.

The operator $h_{F_{4}}^{(t)}$ presents the algebraic form of the $H_{F_{4}}^{(t)}$ Hamiltonian. It preserves the same flag of spaces of polynomials $\mathcal{P}^{\left(F_{4}\right)}$ as in the rational case $(1,2,2,3)$ (now in variables $\left.\tau_{1,3,4,6}\right)$. But it is NOT triangular with respect to the action on monomials $\tau_{1}^{p_{1}} \tau_{3}^{p_{3}} \tau_{4}^{p_{4}} \tau_{6}^{p_{6}}$ in variance to the general statement made in [6]. Making a singular in $\beta$ transformation preserving the flag

$$
\rho_{1}=\tau_{1}, \quad \rho_{3}=\tau_{3}-\frac{1}{8} \beta^{-2} \tau_{1}^{2},
$$




$$
\rho_{4}=\tau_{4}-\frac{3}{16} \beta^{-4} \tau_{1}^{2}, \quad \rho_{6}=\tau_{6}-\frac{3}{4} \beta^{-2} \tau_{1} \tau_{4}+\frac{3}{64} \beta^{-6} \tau_{1}^{3},
$$

we arrive at the $F_{4}$ trigonometric Hamiltonian $h_{F_{4}}^{(t)}(\rho)$ in triangular form. From this form we can immediately calculate the spectrum:

$$
\begin{aligned}
E_{n}= & 4\left[p_{1}\left(p_{1}+2 p_{3}+3 p_{4}+4 p_{6}\right)+2 p_{3}\left(p_{3}+2 p_{4}+3 p_{6}\right)+p_{4}\left(3 p_{4}+8 p_{6}\right)+6 p_{6}^{2}+\right. \\
& \left.\nu\left(5 p_{1}+6 p_{3}+9 p_{4}+12 p_{6}\right)+2 \mu\left(3 p_{1}+5 p_{3}+6 p_{4}+9 p_{6}\right)\right] \beta^{2}+ \\
& 4 \beta^{2}\left(7 \nu^{2}+14 \mu^{2}+18 \nu \mu\right), \quad p_{i}=0,1,2 \ldots,
\end{aligned}
$$

Since we know the flag of invariant subspaces, we can calculate eigenfunctions by linear algebra means. It is worth mentioning that in [7] it was found nonminimal flag $(1,2,3,4)$.

\section{Acknowledgments}

This work is supported in part by the grant IN124202 (UNAM).

\section{References}

[1] K.G. Boreskov, J.C. Lopez V. and A.V. Turbiner, "Solvability of $F_{4}$ integrable system",

Int.Journ.Mod.Phys. A16, 4769-4801 (2001) hep-th/0108021

[2] M. A. Olshanetsky and A. M. Perelomov, "Quantum integrable systems related to Lie algebras",

Phys. Rep. 94 (1983) 313

[3] W. Rühl and A. V. Turbiner, "Exact solvability of the Calogero and Sutherland models",

Mod. Phys. Lett. A10 (1995) 2213-2222

[4] M. Rosenbaum, A. Turbiner and A. Capella, "Solvability of the $G_{2}$ integrable system",

Intern.Journ.Mod.Phys. A13, (1998) 3885-3904 solv-int/9707005

[5] O. Haschke and W. Ruehl, "Is it possible to construct exactly solvable models ?",

Lect.Notes Phys. 539 (2000) 118-140 hep-th/9809152

[6] S.P. Khastgir, A.J. Pocklington, R. Sasaki, "Quantum Calogero-Moser Models: Integrability for all Root Systems", J.Phys. A33 (2000) 9033-9064 hep-th/0005277

[7] O. Haschke and W. Ruehl, "The construction of trigonometric invariants for Weyl groups and the derivation of corresponding exactly solvable Sutherland models",

Mod. Phys. Lett. A14 (1999) 937-949 math-ph/9904002 